\title{
Association between Socio-demographic Characteristics of Community Health Workers with Compliance to Quality Delivery of Integrated Community Case Management of Febrile Illness services in Nigeria
}

\author{
Article by Bright Orji ${ }^{1}$, William Brieger ${ }^{2}$, Lindiwe Innocentia ${ }^{3}$ \\ ${ }^{1,3}$ Texila American University \\ ${ }^{2}$ Public Health, the Johns Hopkins Bloomberg \\ Email: bright2orji@yahoo.com ${ }^{1}$
}

\begin{abstract}
The objective of the study was to investigate the association between community health workers (CHWs) socio-demographic characteristics with compliance to quality delivery of integrated community case management (iCCM) of malaria, diarrhea and simple respiratory infection. This was an implementation research study conducted in two local government areas of Akwa Ibom State, Nigeria with a total of 152 CHWs. The study used an observational data collected by the ministry of health and implementing agency in 2010. The observational data was re-analyzed using SPSS version 17 to examine the association between the dependent variable, quality improvement performance score and independent variable - socio-demographic characteristics of CHWs. $21 \%$ of the CHWs were male while $79 \%$ were female CHWs. The CHWs overall mean age was 36.8( \pm 8.7$)$ years. The CHWs were assigned to the following age groups: <36 years (53\%), and >36 years (47\%). $12.0 \%$ of the CHWs had primary education; $74.0 \%$ attained secondary school while $14.0 \%$ of the CHWs attained postsecondary school. $63.0 \%$ of the CHWs were unskilled while 37.0\% of the CHWs were skilled. Chisquare test result revealed that $81.5 \%(n=124)$ of the $152 \mathrm{CHWs}$ studied were compliant to quality improvement performance. Logistic regression analysis showed that socio-demographic characteristic associated with quality improvement performance was sex (OR 2.717, 95\% CI: 1.064-6.937, $p=0.037$ ). To introduce and sustain the impact on quality performance among CHWs requires training, supportive supervision and strong coordination.
\end{abstract}

Keyword: So-demographic characteristics, Community health workers, integrated community case management of malaria, pneumonia and diarrhea; quality improvement performance standards.

\section{Introduction}

Evidence based interventions to prevent malaria deaths are available. The World Malaria Report 2016 revealed that $74 \%$ of global suspected malaria cases were tested using RDTs with greater number of proportion taking place in the public health sector. Malaria treatment using ACTs increased from $29 \%$ in 2010 to $80 \%$ in 2015 representing almost a three-fold increase. These achievements were as results of countries engaging community health workers (CHWs) in order to mitigate the challenges associated with lack of human resource for health. Training CHWs to provide quality of malaria care and deploying them to work at the community under the supervision of the frontline health care workers has helped in taking essential life-saving interventions to the door-step of those who need them. This approach was made popular by the African Program for Onchocerciasis Control (APOC) that used community directed treatment with invermectin (CDTI). The approach helped to make substantial contributions to the control and treatment of Onchocerciasis (York KJ., et al., 2015). Therefore understanding the factors influencing CHWs contributions, and compliance to performance standards will help sustain the gains of their involvement in the delivery of quality of care.

In Ghana, Edgar Necochea et al (2015) demonstrated that application of provider performance quality improvement assessment to community-based health services in 2011-2014 among 61 community health programs (CHPs) resulted in well over 30\% improvement from baseline within one year of program implementation. In Zambia, provider performance of quality improvement standard was introduced to the lay workers on the delivery of PMTCT counseling and group education sessions. This excluded clinical services, and the study concluded that the PMTCT lay workers contributed to the increase of clients flow and work-load of the health workers by taking off the responsibility and freed 
up time for them to engage in other duties (Young MI Kim, et al 2013). Similarly, studies on taskshifting strategies that involved CHWs have reported high impact and impressive contributions of CHWs on taking responsibilities of counseling and health education sessions off the health workers (Born LJ , et al 2012; Morris MB., et al 2009). These evidences have continued to support active involvement and participation of CHWs in public health interventions as part of the key strategy to reduce the effect of critical shortages of health workers in the developing countries. In rural Nepal, female community health volunteers were able to provide satisfactory quality performance in maternal and child health services (Dilaram Acharya, et al, 2016). In another effort, community health volunteers in Malaysia were able to understand their roles, and commitment that led to high quality improvement of performance standards when they were engaged on early detection of Non-communicable diseases, improving knowledge to healthy behavior and life-style (Liang Chung HM.., et al 2017).

In one study in Nigeria, Orji B., et al shows that $97.6 \%$ of the female volunteer CHWs were adherent with malaria treatment protocol compared to $89.0 \%$ of the male. The authors concluded that women are more disposed to responding to volunteer job and may not be unconnected to their human nature especially given their societal roles and responsibilities in the family. Similarly, this finding was collaborated by Dilaram Acharya et al (2016) that reported that quality improvement performance of female community health volunteers are affected by some of their socio-demographic characteristics. Bagonza J., et al, 2014 in rural Uganda demonstrated that performance of female CHWs was high in the delivery of maternal and child services; and this collaborated an earlier work carried out by Alam $\mathrm{K}$, Tasneem S and Oliveras E in rural Bangladesh in 2012. Another study in Zambia, Hamer DH et al, 2012, found female CHWs more compliant than facility based health workers in following RDTs positive results to dispense ACTs according to treatment protocols. The authors concluded that female CHWs high performance is associated with the ease other women may have in seeking care from among their peers; and also male CHWs area distracted by their societal roles of providing the needs of their family members which might make them less effective to unpaid jobs.

However, there are studies that demonstrated that gender did not influence performance of CHWs. Example, one study in Zambia Harvey SA., et al, 2008 that trained CHWs on the use of RDT to test for malaria compared along the line of those that use job-aids, manufacturers manuals etc. concluded that gender was not statistically significant with quality performance in the delivery of malaria services. Especially when compared on the use of RDT to correctly test for malaria, result interpretations and appropriate dispensing of ACTs.

In another dimension the study found volunteers who were in age brackets older than 36 years more adherent with appropriate and correct treatment protocols compared with those younger than that age bracket. Similar findings have been reported else-where, in one study in the United States volunteers were highest in number with people between age bracket 35 - 44 years compared with those under 25 years (13\%) and 65 years or more (18\%) (Hayghe HV., 1991). Some studies have found that age of the CHWS is associated with their performance. In one study, Melvin Hsien et al 2017, demonstrated that older CHWs are more concerned with the delivery of altruistic services whereas the younger CHWs are easily distracted by career concern, spouse and peer pressure that make them not so effective. However, in Guinea-Bissau Lopez SC., et al 2014 found younger CHWs to deliver more quality performance in the management of diarrhea among under-five children. This findings collaborate the result of the work by Dilaram Acharya et al 2016 that reported age of the CHWs as not a predictor of knowledge and performance. Earlier work in urban slum of Dhaka by Alam K., et al 2012 has shown that attrition among younger CHWs may make them less effective in quality improvement of performance standards. Melvin Hsien et al 2017 has noted high attrition of younger CHWs from service, and opined that if this was not addressed it would lead to critical shortfall in the number of CHWs available for volunteer services.

Also, available evidence shows that generally education impacts on volunteerism. People with higher level of education are more likely to participate in volunteer job compared to those with lower education. Liang Chung ML., et al 2017, noted that education is a positive factor that could influence CHWs to perform much better and improve quality of services in the community where they serve. Similarly, Dilaram Acharya et al, 2016 opined that the higher educational level that the CHWs have, the more knowledge they have and complaint to guidelines and set of performance standards. The study 
in the US by Hayghe HV., (1991) shows that proportion of volunteers was more among people with college education compared to those with lower qualification. In Nigeria, Orji B. et al 2010 reported too that proportion of volunteers were more among those with higher education. However, education was not found to significantly impact compliance with treatment protocols.

The study also reported that proportion of people with jobs or vocations were more likely to volunteer their services than those without jobs. This is because vocations or jobs will support them to provide for their family members, and the opportunity to take unpaid jobs. This has been collaborated by study in Kuching district of Sarawak Malaysia where job occupations of the CHWs were not predictor of role performance and did not influence service outcome.

\section{Materials and methods}

An implementation research with measurement over time specifically designed to measure independent variable- CHWs socio-demographic characteristics and dependent variable - compliance with quality improvement of performance standards. Performance standard is defined as taking history/examination; conducting RDTs and appropriate management of febrile illness. One page checklist was developed by the project to monitor and measure the performance of the CHWs. The checklist has 37 performance criteria (PC) that was used to observe and measure CHW's knowledge, skills and competence in 3 sections: History taking and Examination; Conducting RDTs for Malaria; and Illness Management. Each section of the performance criteria has between 11 to 13 verifiable criteria. The checklist was used to train the CHWs, and also used to observe them while providing services at the community; each correctly performed criterion was scored 1 point. A score $\geq 80 \%$ was set as compliant based on implementing agency's participant's pass mark at trainings; while $\leq 80 \%$ is adjudged as non-compliant. Four rounds of assessments were conducted after the baseline at an interval of two months between project implementation periods June 2011 - March, 2012.

The study population was the $152 \mathrm{CHWs}$ selected by the communities. The CHWs were selected by the communities using agreed criteria. These criteria include residency in the community where they served, able to read and write, willingness to volunteer services, approved by spouse if married, atten d monthly meetings and submit reports. The community held - community meetings with men, women, youths and visitors residents in the community in attendance. In some communities there were people that volunteered their services and if more than the number required, the communities conducted voting exercise to select appropriate numbers of CHWs that will be able to serve the community. In some other communities, selection was based on previous experience especially communities that have had experience with onchocerciasis where CHWs were in existence. At the end, twenty-one percent of the CHWs were male, while seventy-nine were female. The mean age of the CHWs was 36.8( \pm 8.7$)$ with highest educational level as senior secondary school. The vocations of the CHWs include farming, trading, retirees, civil servants etc. The CHWs provided services to 2,206 over one year period. The CHWs records were audited at the end of twelve calendar months.

The study used purposive sampling technique because all the data elements were included. All the 152 trained CHWs were sampled to investigate the quality of improvement performance standards in the delivery of integrated community case management of malaria, pneumonia and diarrhea services.

\section{Data management}

The study focused on data that was used to answer research questions based on ascertaining the extent to which the socio demographic characteristics measured in sex, age, education and vocation impacts on the CHWs performance. Chi-square tests was conducted at 0.05 level of significance, and corresponding p-values reported to three decimal places with values less than (0.001) reported as $(<0.001)$. Statistical decision to reject the null hypothesis was based on the following:

- $P$-value less than or equal to the alpha level, reject the null hypothesis and accept the alternative hypothesis

- $P$-value greater than the alpha level, retain the null hypothesis and reject the alternative hypothesis. The research question of the study is: 
RQ1: To what extent does socio-demographic characteristic as measured by sex, age, education and vocations make a difference on CHWs' compliance to quality improvement of performance standards scores for integrated community case management of malaria, pneumonia and diarrhea?

Ho1: CHW's socio demographic characteristics (sex, age, educational levels and vocation) do not make a difference in quality performance scores for integrated community case management of malaria, pneumonia and diarrhea.

Ha1: CHW's socio demographic characteristics (sex, age, educational levels and vocation) do make a difference in quality performance scores for integrated community case management of malaria, pneumonia and diarrhea.

Chi-square test for association was conducted to ascertain the extent to which social demographic characteristic status as measured by sex, age, education and vocations. Logistic regression results is used to determine the level of significance and the decision to reject or retain the null hypothesis based on the stated alpha level of 0.05 .

\section{Results}

\section{Socio-demographic characteristics}

One hundred and fifty-two (152) CHWs were studied. $21 \%$ were male while $79 \%$ were female CHWs. The CHWs overall mean age was $36.8( \pm 8.7)$ years. The mean age for the male CHWs was 37.6 $( \pm 4.3)$ years while the mean age for the female CHWs was $36.6( \pm 9.5)$ years. The youngest age of the study CHWs was 30 years while the oldest age was 49 years old with a range of 30-49. The CHWs were assigned to the following age groups: $<36$ years, and $>36$ years. Eighty (80) or $53.0 \%$ of the CHWs were in the group < 36 years while seventy-two (72) or $47 \%$ of the CHWs were in the group of $>36$ years old. Eighteen (18) $12 \%$ of the CHWs had primary education. One hundred and thirteen (113) of the CHWs or $74.0 \%$ attained secondary school while twenty-one (21) or $14.0 \%$ attained postsecondary education. Ninety-six (96) or $63.0 \%$ of the CHWs were unskilled while 56 of the CHWs or $37.0 \%$ of the CHWs were skilled. Most of the CHWs were unskilled $n=96$. Table 2 below provides information about the occupation of the studied CHWs.

A Chi-square test analysis of independent and dependent variables revealed that in overall, a total of $81.5 \%(\mathrm{n}=124)$ of the CHWs were compliant to performance standards. These include $69.0 \%(\mathrm{n}=22)$ of the male and $85.0 \%(n=102)$ of the female CHWs. The result of the Chi-square test analysis indicated that sex was significantly associated with compliance to quality performance standards $(\mathrm{p}=0.035)$. Of those, $81.5 \%(n=124)$ studied CHWs were compliant to quality performance standards; $80.0 \%(n=64)$ were within the age brackets of $\leq 36$ years while $83.0 \%(n=60)$ were CHWs $>36$ years old. The result of the Chi-square test analysis shows that age was not significantly associated with compliance to quality performance standards $(\mathrm{p}=0.596)$. CHWs with primary educational level that were compliant included $72.0 \%(\mathrm{n}=13)$, secondary educational level $82 \%(\mathrm{n}=93)$ while post-secondary educational level $86.0 \%(\mathrm{n}=18)$. Educational level was not significantly associated with compliance to quality improvement of performance standards $(\mathrm{p}=0.515) .83 .0 \%$ of the unskilled CHWs was compliant to quality improvement performance standards $(n=80)$ compared $79 \%$ of the skilled CHWs $(n=44)$. CHWs vocation was not significantly associated with $\mathrm{CHW}$ 's compliance to quality improvement performance standards for integrated community case management of malaria, pneumonia and diarrhea $(\mathrm{p}=0.465)$.

The result of the logistic regression analysis showed Hosmer and Lemeshow (model of goodness fit test) fits the data $\left(\mathrm{X}^{2}=22.165 ; \mathrm{df}=6 ; P=0.001\right)$, and statistically significant which correctly explains the $8.20 \%$ (Nagelkerke $\mathrm{R}^{2}$ ) of the variance in compliance and correctly classified $81.6 \%$ of the performance. The CHWs' socio-demographic characteristic sex and compliance to quality improvement performance standards results in changes in quality of performance standards (defined as female and male by CHWs delivery of quality performance. The odds ratio for sex was (OR 2.717, 95\% CI: 1.064-6.937, $\mathrm{p}=0.037$ ); age measured by $\leq 36$ years old and $>36$ years old CHWs (OR 0.568, 95\% CI: $0.221-1.458, p=0.240$ ); Educational level measured by primary, secondary and post-secondary (OR 0.685, 95\% CI: 0.2671.761, $\mathrm{P}=0.433$ ); and vocation as measured by skilled and unskilled CHWs (OR 0.729, 95\% CI: 0.308 $1.728, \mathrm{p}=0.473)$. Sex predicted compliance among the female and male CHWs to quality improvement performance standards in Akwa Ibom State, Nigeria at a statistically significant level ( $\mathrm{c}<=0.05$ ). Therefore, the null hypothesis that CHW's socio demographic characteristics sex as measured by female 
and male do not make a difference in quality performance scores for integrated community case management of malaria, pneumonia and diarrhea could not be rejected.

\section{Discussions}

The socio-demographic characteristics sex of the CHWs alone varies with complaint to quality improvement performance. Female CHWs were found to be more complaint compared to their male counter-parts. This study's findings also indicated that as the rounds of measurement of quality improvement performance standards, and feedback provided to the CHWs increases, compliance to performance standards of the CHWs improves. Addressing the barriers that limits the extent of impact on compliance with performance standards may improve compliance rates, quality of iCCM services provided by $\mathrm{CHWs}$ and decrease high mortality and morbidity due to malaria, pneumonia and diarrhea. Female CHWs performance showed $85 \%$ compliance compared to male CHWs of $69 \%$. The more the length of services provided, the female CHWs improve their services. This result shows that female CHWs is 2.7 times more likely to provide quality integrated community case management of malaria, diarrhea and pneumonia than male counter-parts. This finding deferred with what was earlier reported in a study conducted in Zambia where CHWs gender was not statistically significant with quality service delivery of malaria services especially on the use of RDT to correctly test for malaria and dispense ACTs appropriately (Steven Harvey, Larissa Jennings and Chinyama et al, 2008). The report indicated that more women in the community are care givers of under-five children, and therefore peers with the female CHWs. It is easier for the female CHWs to bond with the community women, improve friendship, communication; and promote service utilization. The female CHWs could check for the expiry dates of the RDTs, collect adequate blood from the clients and provide correct interpretation of the results as well as dispense ACTs following RDTs results. These were areas that seem difficult for some of the male CHWs. This corroborates earlier study findings where the performance of female CHWs was high in the delivery of maternal and child health services in Uganda and Bangladesh that share similar characteristics with Nigeria (James Bagonza, Simon PS Kibira, and Elizeus Rutebemberwa, 2014; Alam K, Tasneem S, Oliveras E., 2012); and another study in rural Zambia that found CHWs more compliant than facility based health workers in dispensing ACTs following RDTs positive results (Davidson H. Hamer, Erin Twohig Brooks, Katherine Semrau et al, 2012). The authors attributed the reason for female CHWs high performance over the male to the comfort women may have in seeking care from women volunteer care givers as well as to the societal role of men that might affect level of performance. This role include men's social responsibility to their families i.e. for providing the needs of their families. This role might make male CHWs not to be effectively disposable to unpaid job.

Data analysis using the Chi-square test revealed that there was no association between age and compliance to quality improvement performance standards ( $\mathrm{p}$-value $=0.596$ ). Though the results showed that as service delivery improves during every round of assessments, more of the CHWs in the age brackets $>36$ performed better than the younger ones with $83 \%$ compared to $80 \%$. This corroborates other studies where best role performance was associated with CHWs from the age brackets of 35 to 44 years old. Melvin Hesien Liang Chung et al, 2017 explained that CHWs who are older appear more interested in "values function" especially in making more people happy. Logistic regression was conducted to further test for possible influence of age as a socio-demographic characteristics with compliance to quality improvement performance standards. The Hosmer and Lemeshow model explains $8.20 \%$ of the variance in compliance and correctly classified $81.6 \%$ of the performance cases while the odd ratio of age measured by $\leq 36$ years old and $>36$ years old CHWs (OR 0.568, 95\% CI: 0.221-1.458, $\mathrm{p}=0.240$ ). Therefore, there is no difference in compliance to the quality improvement performance among the CHWs. However, contrary to our study, younger CHWs have been found to more accurately provide correct management of diarrhea among children in Guinea-Bissau (Sergio C Lopez, Antonio J Cabral and Bruno de Sousa, 2014). This study's findings supports the results from an earlier study where the age of the CHWs was not a predictor on knowledge and performance even when ages of the CHWs varied from <35 years to >45 years old (Dilaram Acharya, Jitendra Kumar Singh, Samaj Adhikari and Varidmala Jain, 2016). In another study, Khurshid Alam and Elizabeth Oliveras 2014 reported that younger CHWs <25 years in the Urban slum of Dhaka were found to be more in attrition 
by leaving the slum compared to older ones hence had poor retention in the job and less effective in performance. This is because younger CHWs appear to be easily influenced by their peers; and distracted by either their spouse, career social than "value functions" (Melvin Hsien Liang Chung, Helmy Hazmi, and Whye Lian Cheah, 2017). Interestingly, the CHWs that participated in the intervention in Akwa Ibom State, Nigereia were more of female CHWs and large proportion of them were married. The project implementation report demonstrated that agreement with their spouse were sought by the community prior to selection, and this may have contributed to their stability in the job, and improved service utilization as observed. Also, the report emphasized the monthly community health workers' meetings with their supervisors which was used to track quality improvement efforts using tools based on set performance criteria and coordinate monthly reporting system among the CHWs. This approach may have strengthened coordination among CHWs, institutionalized process to improve quality improvement across all ages. However, another study showed increase in nonadherence to treatment protocol among cases of maternal death in 2002-2004 compared with 1999-2001 though this was not statistically significant but enough concern for quality of care.

This result demonstrates that educational level as sociodemographic characteristics of the CHWs does not affect or predict compliance with quality improvement standards. The performance of the CHWs with primary education may have been influenced by trainings and supportive supervision provided across CHWs with all levels of educational qualifications. This corroborates the results of an earlier study. Davidson H. Hamer et al, 2012 reported that trainings, supportive supervision and logistics provision were able to strengthen the capacity of CHWs to appropriately conduct RDTs, interpret results, and with good adherence to WHO treatment protocols providing evidence that CHWs can safely implement the new guidelines. One study in Guinea-Bissau demonstrated that CHWs were capable to understand theoretical concepts presented during training, and such knowledge contributed to their performance. The study also opined that the number of trainings attended by CHWs was compared with accuracy in carrying out diagnosis and treatment, the authors could not identify any trend. Besides, one of the statistical test conducted showed that CHWs with less training, and experience performed better with CHWs that had higher number of trainings. The authors concluded that selection of appropriate volunteers as CHWs, supportive supervision and continuous trainings should be given the same level of importance to the training itself (Sergio C Lopez, Antonio J Cabral, and Bruno de Sousa, 2014).

This conclusion supports public health practitioner's policy makers, program managers, service providers and communities in the development of interventions that in considering educational background as a factor and predictor of compliance to quality delivery of services, selection of appropriate persons as CHWs, supportive supervision and trainings should be given top priority. The findings may also assist stakeholders in modification of health program interventions that volunteers should not only be given to people with high level education but ensure that adequate training package is provided, and guidelines used in providing training to those that volunteered their services. This will help to boost their compliance with quality improvement performance. Public health providers and other personnel involved in the provision and facilitation of health care services in the community should focus also on interventions such as improving the educational level of volunteers with lower educational level in the form of adult education which could serve as an incentive to motivate their interest and improve their compliance with quality services delivery. According to Allen et al. (2014), community play an important role in community health because they provide both social and health support to their members through health education programs in the Parishes and routinely provide members with current information about different diseases.

This findings showed that vocation is not a predictor of compliance to quality improvement of performance standards for CHWs in Akwa Ibom state, Nigeria. This corroborates earlier study in Kuching district of Sarawak Malaysia where job occupations of the CHWs were not predictor of role performance and did not in any way influence service outcome. As noted earlier, the result of this study might have been influenced by the level of supportive supervision provided by the frontline health workers as well as the training of the CHWs. Supportive supervision has been identified as one of the critical component of the work of Community services. Melvin Hsien et al has noted that supervision of the CHWs will improve and impact positively on their performance. These features - training and supportive supervision of community directed interventions may have contributed to the performance 
of the CHWs across all levels of vocations - skilled and unskilled by ensuring compliance to performance standard especially the monthly meetings of the CHWs that was used to address gaps, and support the CHWs improve their performance.

\section{Conclusion}

Compliance to quality improvement of performance standards remains both a national and global public health concern due to increasing use of community health volunteers to support service delivery. This has been brought about by the critical challenges with poor availability of human resource for health. Also, the emergence of community approach in the delivery of iCCM to complement integrated management of childhood illness (IMCI) which is facility based has thrown up the challenges of dependence on the work of the CHWs alongside of quality service delivery. Therefore, lack of compliance with quality improvement of performance standards and socio-demographic characteristics, features of CDI organizational structures may continue to hinder efforts to improve best practices and quality improvement among the community health workers if not addressed. Future studies should focus on the impact of covariates such as income range, marital status, perceived community status, and previous experience with health work on compliance with quality improvement of performance standards.

\section{Reference}

[1]. Alam K., Tasneem S., Oliveras E. (2012). Retention of female volunteer community health workers in Dhaka urban slums: a case-control study. Health Policy Plan 27(6): 477-86. Doi: 1093/heapol/czr059.

[2]. Bagonza J., Kibira PS., Rutebemberwa E., (2014). Performance of health workers managing malaria, pneumonia and diarrhea under community case management program in central Uganda: a cross sectional study. Malaria Journal doi: 10.1186/1475-2875-13-367.

[3]. Born LJ, Wamulume C, Neroda KA, Quiterio N, Gigant MI, Morris M, Bolton-Moore C, Baird S, Sinkamba M, Tropp SM, Reid SE (2012). Evaluation of a task-shifting strategy involving peer education in HIV Care and treatment clinics in Lusaka, Zambia. Journal of Public Health in Africa.

[4]. Dilaram Acharya, Jitendra Kumar Singh, Samaj Adhikari, Varidmala Jain (2016). Association between sociodemographic characteristics of female community health volunteers and their knowledge and performance on maternal and child health services in Nepal. Journal of Multidisciplinary Healthcare 9:111-120.

[5]. Edgar Necochea, Vandana Tripathi, Young MI Kim, Nabeal Akran, Yoland Hyjazi, Maria da Luz Vaz, Emmanuel Otolorin, Tsigue Pleah, Tambudzai Rashidi, Dustan Bishanga (2015).

[6]. Hayghe HV. (1991). Volunteers in the US: Who donates time? Monthly Labor Review.

[7]. Hamer DH., Brooks ET., Semrau K., Pilingana P., MacLeod WB., Siazeele K., Sabin LL., Thea DM., Yeboah-Antwi (2012). Quality and safety of integrated community case management of malaria using rapid diagnostic tests and pneumonia by community health workers. Pathog Glob Health. 106(1): 32-39. doi: 10.1179/1364859411Y.0000000042Harvey S.A, Jennings L, Chinyama M, Masaninga F, Mulholland K, Bell DR, 2008. Improving community health worker use of malaria rapid diagnostic tests in Zambia: package instructions, job aid and job aid-plus-training. Malar J 7: 160.

[8]. Implementation of Standard-Based Management and Recognition approach to quality improvement in maternal, newborn, and child health programs in low-resource countries. International Journal of Gynecology and Obstetrics 130 S17-S24.

[9]. Lopes SC., Cabral A., de Sousa B., (2014). Community health workers: to train or to restrain? A longitudinal survey to assess the impact of training community health workers in the Bolama Region, Guinea-Bissau. Hum Resour Health. 12:8 doi: 10.1186/1478-4491-12-8.

[10]. York KJ, Kabole I., Mrisho M., Berry DM. Schmidt E., (2015). Factors Affecting Community Participation in the CDTI Program in Morogor, Tanzania. Journal of Nursing Scholarship vol. 47, pages 96-104.

[11]. Morris MB, Chapula BT, Chi BH, Mwanza J, Manda H, Bolton C, Pankratz DS, Stringner JS, Reid SE (2009). Use of task-shifting to rapidly scale-up HIV treatment services: experiences from Lusaka, Zambia. BMC Health Services Research.

[12]. Melvin Hsien Liang Chung, Helmy Hazmi, and Whye Lian Cheah (2017). Role Performance of Community Health Volunteers and its Associated Factors in Kuching District, Sarawak. Journal of Environmental and Public health ID 9610928. 
DOI: $10.21522 /$ TIJPH.2013.06.02.Art005

ISSN: $2520-3134$

[13]. Orji BC., Brieger WR., Otolorin EO., Nwadike J., Bassey EV., Nkanga M. (2010). Health System Strengthening through Community Referral in the Management of Febrile Illness in Nigeria, ASTMH annual Meeting, Georgia - Atlanta (Abstract \#1277.

[14]. Young MI Kim, Maureen Chilila, Hildah Shasulwe, Joseph Banda, Webby kanjipite, Supriya Sarkar, Eva Bazant, Cyndi Hiner, Maya Tholandi, Stephanie Reinhardt, Joyce Chongo Mulilo, and Adrienne Kols. (2013). Evaluation of quality improvement intervention of HIV (PMTCT) at Zambia defence force facilities. BMC Health Services Research 13:345.

Table 1. Socio demographic characteristics of the CHWs

\begin{tabular}{|c|c|c|c|}
\hline & \# of CHWs & $\%$ of CHWs & \\
\hline & & & P-value \\
\hline No. of Participants & $(\mathrm{N}=152)$ & $(\%=100)$ & \\
\hline \multicolumn{4}{|l|}{ Sex } \\
\hline Male & 32 & 21.0 & \\
\hline Female & 120 & 79.0 & \\
\hline \multicolumn{4}{|l|}{ Age } \\
\hline Mean age & $36.8 \pm 8.7$ & & \\
\hline Male mean age & $37.6 \pm 4.3$ & & 0.00 \\
\hline Female Mean age & $36.6 \pm 9.5$ & & \\
\hline$<=36$ & 80 & 53.0 & \\
\hline$>36$ & 72 & 47.0 & \\
\hline \multicolumn{4}{|l|}{ Educational Level } \\
\hline Primary & 18 & 12.0 & \\
\hline Secondary & 113 & 74.0 & \\
\hline Post-secondary & 21 & 14.0 & \\
\hline \multicolumn{4}{|l|}{ Occupation } \\
\hline Unskilled & 96 & 63.0 & \\
\hline Skilled & 56 & 37.0 & \\
\hline
\end{tabular}

Participants $=\mathrm{N}=152$

Complaints $(\mathrm{n}=124)$

Non-complaints $(=28)$

Table 2. Chi-square test analysis of independent and dependent variables

\begin{tabular}{|c|c|c|c|c|c|c|c|}
\hline $\begin{array}{l}\text { Socio- } \\
\text { characteristics }\end{array}$ & Compliant & $\%$ & $\begin{array}{l}\text { Non- } \\
\text { compli } \\
\text { ant }\end{array}$ & $\%$ & $\mathrm{X}^{2}$ & Df & p-value \\
\hline \multicolumn{8}{|l|}{ Age (years) } \\
\hline \multirow[t]{2}{*}{$<36$} & 64 & 80 & 16 & 20 & & & \\
\hline & & & & & 0.282 & 1 & 0.596 \\
\hline$>36$ & 60 & 83 & 12 & 17 & & & \\
\hline \multicolumn{8}{|l|}{ Sex } \\
\hline \multirow{2}{*}{ Male } & 22 & 69 & & 31 & & & \\
\hline & & & 10 & & & & \\
\hline Female & 102 & 85 & & 15 & 4.439 & 1 & 0.035 \\
\hline \multicolumn{8}{|c|}{ Educational level } \\
\hline Primary & 13 & 72 & 5 & 28 & & & \\
\hline Secondary & 93 & 82 & 20 & 18 & 1.327 & 2 & 0.515 \\
\hline $\begin{array}{l}\text { Post- } \\
\text { secondary }\end{array}$ & 18 & 86 & 3 & 14 & & & \\
\hline
\end{tabular}


Texila International Journal of Public Health Volume 6, Issue 2, Jun 2018

\begin{tabular}{|l|l|l|l|l|l|l|l|}
\hline Vocation & 80 & 83 & 16 & 17 & & & \\
\hline Unskilled & & & & & 0.534 & 1 & 0.465 \\
\hline Skilled & 44 & 79 & 12 & 21 & & & \\
\hline
\end{tabular}




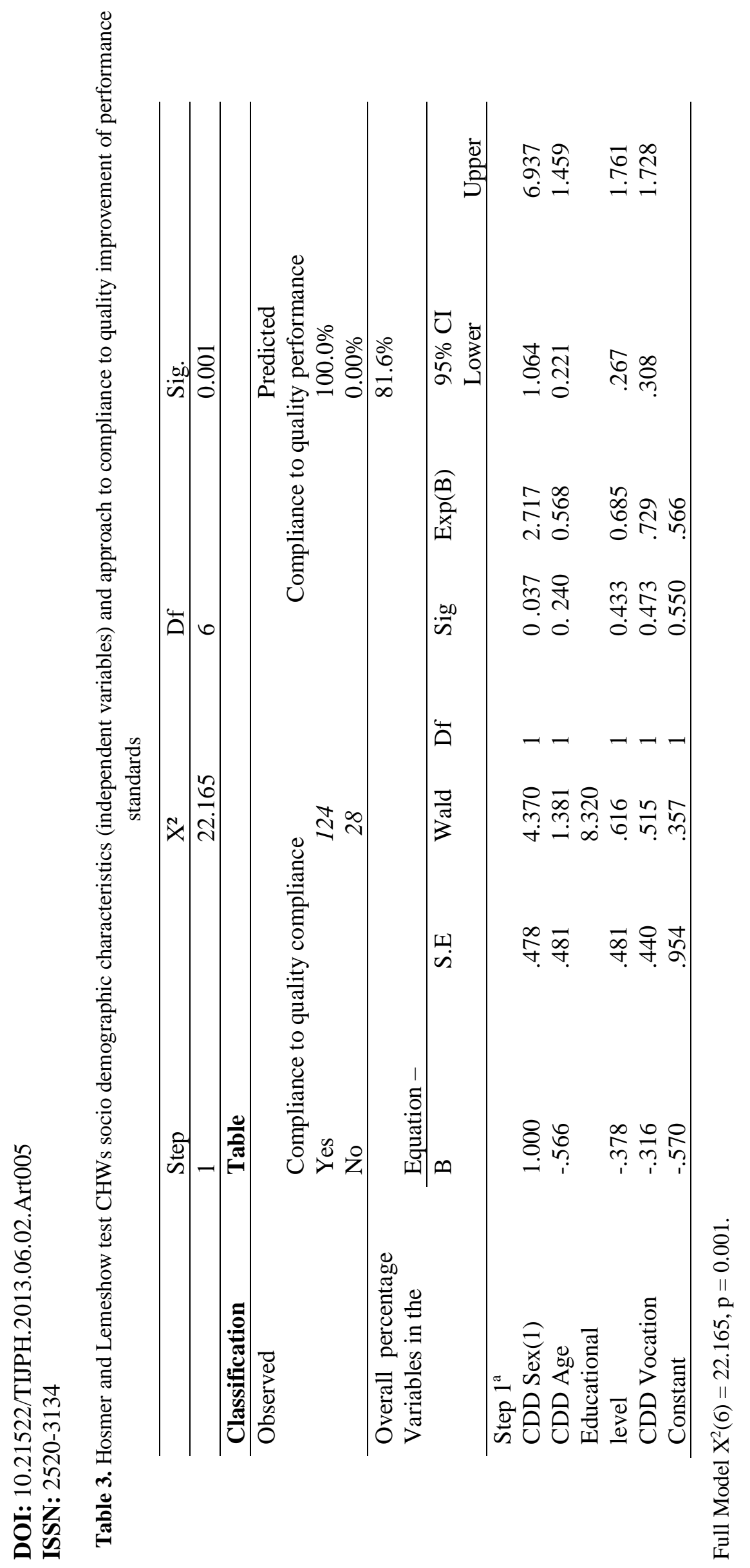

